"C2020 IEEE. Personal use of this material is permitted. Permission from IEEE must be obtained for all other uses, in any current or future media, including reprinting/republishing this material for advertising or promotional purposes, creating new collective works, for resale or redistribution to servers or lists, or reuse of any copyrighted component of this work in other works." 


\title{
Wirelessly Powered Internet-of-Things Sensors Facilitated by an Electrically Small Egyptian Axe Dipole Rectenna
}

\author{
Wei Lin and Richard W. Ziolkowski \\ Global Big Data Technologies Centre, \\ School of Electrical and Data Engineering, \\ University of Technology Sydney, \\ Ultimo 2007, Sydney, Australia, \\ Email: Wei.Lin@uts.edu.au, Richard.Ziolkowski@uts.edu.au
}

\begin{abstract}
Wirelessly powered Internet-of-Things (IoT) sensors have many unique advantages compared with wire-powered or battery-based sensors. This paper presents two wirelessly powered sensors (light and temperature) facilitated by a highly compact and efficient electrically small rectenna. The IoT sensor is integrated into the rectifier circuit of the rectenna, which converts the captured AC power to DC energy. The output DC voltage of the rectifier is dependent on the variable impedance of the sensor corresponding to different discerned values. Once an acoustic alarm is attached to this sensor-augmented rectenna, a certain amount of the measured parameter (light or temperature level) can activate the alarm. The receiving antenna of the system is a modified version of a metamaterial-inspired electrically small Egyptian axe dipole (EAD) antenna. By meandering the length of the driven element, inductive impedance was achieved to directly match the receiving antenna to the capacitive impedance of the rectifier circuit. Prototypes of the wirelessly powered light and temperature sensors were fabricated and measured. Experimental results successfully demonstrated the light and temperature sensing performance. Both systems are highly compact and very thin (diameter is $0.13 \lambda_{0}$ and thickness is $0.002 \lambda_{0}$ ) with a $k a$ value equal to 0.47 . They are excellent representatives of WPT-driven sensors for the emerging wireless IoT applications.
\end{abstract}

\section{INTRODUCTION}

Wirelessly powered Internet-of-Things (IoT) sensors are becoming more and more attractive because of their unique advantages compared with traditional wired-powered or battery-based sensors. For instance, the current wire-powered sensor systems for monitoring the temperature in sewer pipes, as reported in [1], [2], suffer from severe cable corrosion and difficulties in relocating them. On the other hand, wirelessly powered IoT sensors are more flexible, robust and able to operate for a very long time. Such a sewer pipe application is illustrated in Fig. 1.

A wireless power transfer (WPT) rectenna is the key component in any wirelessly powered IoT sensor. Many WPT rectennas have been investigated to date; some typical examples are reported in [3]-[5]. However, it remains challenging to achieve all the desired features of a WPT-based sensor for IoT applications, i.e., simultaneous compact size, high efficiency, and wide wireless power capture capacity.
This paper will present wirelessly powered light and temperature sensors based on a highly compact and efficient sensor-augmented rectenna that achieves all of the above desired features. Prototypes of both systems have been successfully demonstrated. Because of their omnidirectional wireless power capture capacity, the developed IoT sensors are ideal candidates for placement inside sealed objects for monitoring applications.

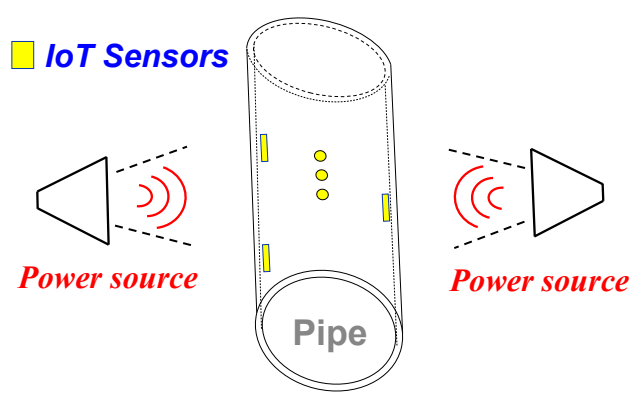

Figure 1. Illustrated wirelessly powered sensor system application.

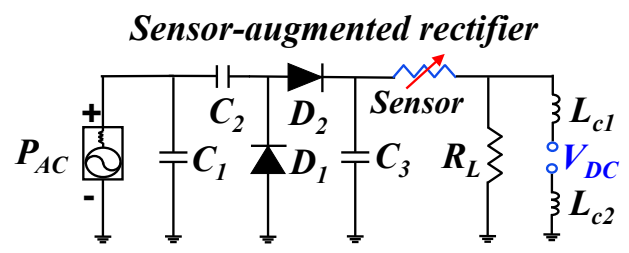

(a)

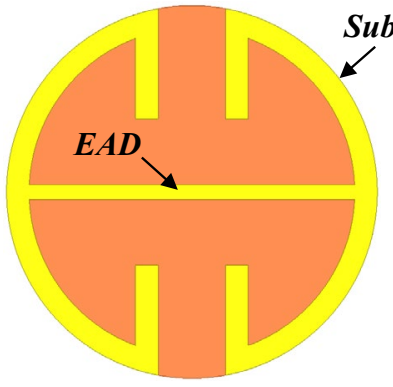

(b)

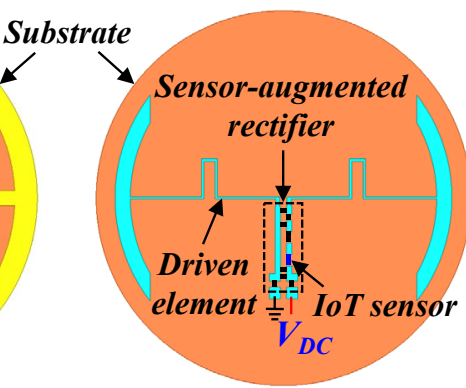

(c)
Figure 2. WPT-driven sensor system. (a) Circuit model of the sensoraugmented rectifier. (b) Top view of the system. (c) Bottom view of the system. 


\section{DeSIGN METhOdOLOGY AND SYSTEM CONFIGURATION}

\section{A. Design of the Sensor-Augmented Rectifier}

The sensor-augmented rectifier is one of the two key parts in this design. As shown in Fig. 2(a), an IoT sensor is integrated into a highly efficient rectifier circuit that has been reported in our previous works [6], [7]. Thus, the output DC voltage $V_{D C}$ is dependent on the impedance of the sensor. For example, a light level can be detected if a photocell augments the circuit, i.e., its impedance varies with the illumination level in an ambient environment. The impedance of a typical photocell is larger than one $\mathrm{M} \Omega$ in dark surroundings, but quickly drops to several $\mathrm{k} \Omega$ in a dim or bright environment. Similarly, the ambient temperature can be detected once a thermistor is incorporated, i.e., it has a temperature-dependent impedance. The light or temperature level is simply represented by the amount of the output DC voltage. A WPT-driven audio light or temperature warning system is realized if an acoustic alarm is attached to the output of the sensor-augmented rectifier.

\section{B. Configuration of the Entire System}

Another key part of these sensor-augmented rectenna systems is the receiving electrically small Egyptian axe dipole (EAD) antenna. It is a modified version of the one reported in our previous works [8] - [10]. As shown in Figs. 2(b) and 2(c), the receiving EAD antenna is highly compact. Its diameter is $0.13 \lambda_{0}$, giving a $k a$ value less than 0.5 . It only requires a single copper-cladded PCB substrate with a very thin thickness $\left(0.002 \lambda_{0}\right)$. Thus, it is easy to fabricate. Excellent broad-angle omnidirectional wireless power capture capacity is demonstrated by its radiation patterns, which are shown in Fig. 3(a). In order to achieve the maximum achievable AC-to-DC conversion efficiency of the rectenna [11], the impedance of the EAD was intentionally modified to be inductive, as shown in Fig. 3(b), in order that it be directly matched to the rectifier. This property was realized by meandering its "driven" element. The impedance becomes inductive when the length of the driven element increases. The rectifier is seamlessly integrated with the EAD antenna to form the entire system.

\section{PERFORMANCE}

The developed wirelessly powered light and temperature sensors were fabricated and tested. Before measuring their sensing performance, the EAD rectenna was tested alone to demonstrate its exceptional AC-to-DC conversion efficiency. As shown in Fig. 4(a), the measured efficiencies agree reasonably well with their simulated values. The peak measured efficiency, $89.9 \%$, is quite close to the simulated value, $87.8 \%$. The measured sensing performance is shown in Fig. 4(b). For the light sensor, the alarm (activation voltage, $0.8 \mathrm{~V}$ ) sounds in a dim and bright environment, but does not in a dark one. For the temperature sensor, the alarm is activated when the ambient temperature exceeds $65^{\circ} \mathrm{C}$. These unique sensing performance characteristics make the developed wirelessly powered sensor systems ideal representatives of WPT-driven sensors for many emerging IoT applications.
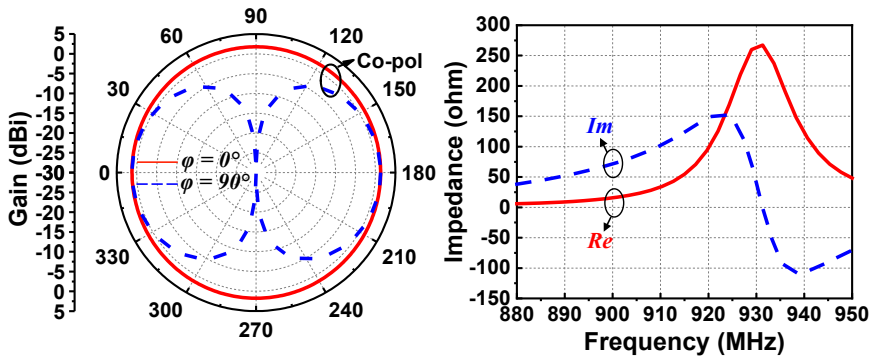

Figure 3. Simulated (a) radiation pattern and (b) input impedance of the driven EAD antenna.
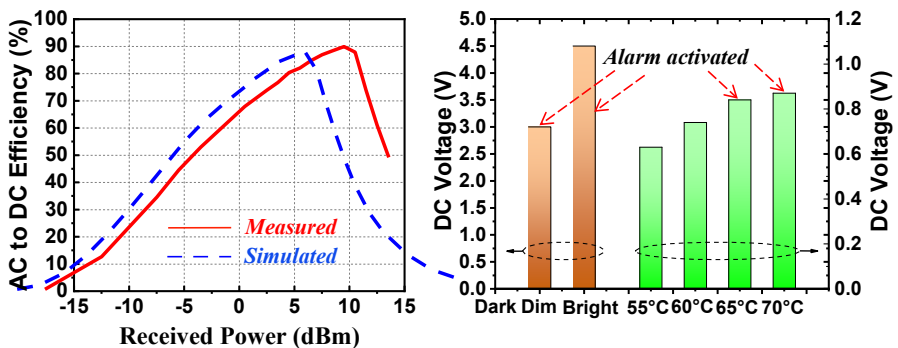

Figure 4. (a) Measured results of the electrically small EAD rectenna; and (b) Sensing performance of the wirelessly powered light and temperature sensors.

\section{REFERENCES}

[1] K. Thiyagarajan, S. Kodagoda, R. Ranasinghe, D. Vitanage, and G. Iori, "Robust sensing suite for measuring temporal dynamics of surface temperature in sewers," Sci Rep., vol. 8, no. 1, 2018.

[2] K. Thiyagarajan, S. Kodagoda, L. V. Nguyen, and R. Ranasinghe, "Sensor failure detection and faulty data accommodation approach for instrumented wastewater infrastructures," IEEE Access, vol. 6, pp. 56 562-56 574, 2018.

[3] A. Massa, G. Oliveri, F. Viani, and P. Rocca, "Array designs for longdistance wireless power transmission: State-of-the-art and innovative solutions," Proc. IEEE, vol. 101, no. 6, pp. 1464 - 1481, Jun. 2013.

[4] Z. Popović, E. A. Falkenstein, D. Costinett, and R. Zane, "Low-power far-field wireless powering for wireless sensors," Proc. IEEE, vol. 101, no. 6, pp. 1397-1409, Jun. 2013.

[5] C. Liu, Y. X. Guo, H. Sun, and S. Xiao, "Design and safety considerations of an implantable rectenna for far-field wireless power transfer," IEEE Trans. Antennas Propag., vol. 62, no. 11, pp. 5798-5806, Nov. 2014.

[6] W. Lin, R. W. Ziolkowski and J. Q. Huang, "Electrically small, highly efficient, Huygens dipole rectennas for wirelessly powering Internet-ofThings (IoT) devices," IEEE Trans. Antennas Propag., vol. 67, No. 6, pp. 3670-3679, June 2019.

[7] W. Lin and R. W. Ziolkowski, "Electrically small Huygens antennabased fully-integrated wireless power transfer and communication system," IEEE Access, vol. 7, pp. 39762-39769, Mar. 2019.

[8] T. C. Baum ; R. W. Ziolkowski ; K. Ghorbani ; K. J. Nicholson, "Electrically small, highly efficient, Huygens dipole rectennas for wirelessly powering Internet-of-Things (IoT) devices," IEEE Trans. Antennas Propag., vol. 65, No. 8, pp. 3827-3837, Aug. 2017.

[9] M. C. Tang, H. Wang and R. W. Ziolkowski, "Design and testing of simple, electrically small, low-profile, Huygens source antennas with broadside radiation performance," IEEE Trans. Antennas Propag., vol. 64, no. 11, pp. 4607-4617, Nov. 2016.

[10] W. Lin and R. W. Ziolkowski, "Electrically-small, low-profile, Huygens circularly polarized antenna," IEEE Trans. Antennas Propag., vol. 66, no. 2, pp. 636-643, Feb. 2018.

[11] W. Lin and R. W. Ziolkowski, "Electrically small Huygens CP rectenna with a driven loop element maximizes its wireless power transfer efficiency," IEEE Trans. Antennas Propag., under revision. 